\title{
EDITORIAL
}

\section{Human papillomavirus (HPV) vaccines: prospects for eradicating cervical cancer}

\section{Introduction}

One of the most important developments of the past 25 years in cancer biology has been the evidence that infection with human papillomaviruses (HPVs) in the lower genital tract is the cause of virtually all cases of cervical carcinoma in women and a smaller, less defined fraction of vulvar, vaginal, anal and penile cancers. HPVs are members of a large family of viruses that infect squamous epithelial surfaces: the 35 HPV types that infect the genital tract fall into two discrete groups:

- Low-risk, non-oncogenic types 6 and 11 and their relatives that cause anogenital warts and are rarely detected in malignant disease.

- High-risk, oncogenic HPVs (hrHPV) types 16, 18, 31, $33,35,45,52,56$ plus about eight other minor types. These can be detected in almost $100 \%$ of cervical cancer biopsies and more than $90 \%$ of the high-grade, cervical intra-epithelial neoplasia (CIN2/3) precursor lesions.

The causal link between HPV infection and cervical cancer has now been established beyond reasonable doubt. ${ }^{1}$ HPV 16 is the most frequently detected HPV in cervical cancers (about 50-60\%) with HPV 18 (10-12\%) being the second most common.

The implication of this is that intervention against this viral infection should prevent the vast majority of cervical cancer cases worldwide. Vaccines are the traditional costeffective means to prevent microbial- and viral-induced diseases: HPV should be no exception and, indeed, substantial progress has been made in the past decade in the development of vaccines to prevent and/or treat these infections. Prophylactic HPV vaccines are in large Phase III trials, and several Phase II trials of therapeutic vaccines for the treatment of established HPV-induced intraepithelial disease are either complete or in progress.

\section{Prophylactic vaccines}

The rationale for prophylactic HPV vaccines is that they induce the production of neutralising antibody to the virus and thus prevent infection on subsequent exposure. In lowrisk HPV infections and natural infections in animals there are serum responses to virus coat proteins in individuals who are or who have been infected; in animal models such individuals are protected against subsequent viral challenge. In these cases, neutralising antibody is generated against determinants on the L1 protein (the major coat or capsid protein) exposed on the outer surface of the intact virus. A vaccine generating such responses must therefore contain L1 protein in the correctly folded tertiary or 'native' form. This is technically very difficult to achieve, but eventually it was shown that the L1 protein, when expressed by vectors such as recombinant baculovirus or yeast, self assembles into virus-like particles (VLPs). The L1 VLP is a conformationally correct, empty capsid (i.e. it contains no DNA) that appears morphologically identical to, and contains the major neutralising epitopes of, the native virion. These VLPs are obvious vaccine candidates, and in Phase I studies in volunteers L1 VLPs were shown to be immunogenic, generating high titres of anti-L1 neutralising immunoglobulin $\mathrm{G}$ ( $\mathrm{IgG}$ ).

Two L1 VLP vaccines are now in Phase III trials: a bivalent HPV 16/18 VLP vaccine developed by GlaxoSmithKline (GSK) and a quadrivalent HPV
16/18/6/11 vaccine from Merck Vaccines. The preliminary efficacy data from Phase II proof-of-principle trials for VLP vaccines are immensely encouraging. A double-blind, placebo-controlled efficacy study of a yeast-derived HPV 16 L1 VLP vaccine developed by Merck was published in 2002. All vaccinees in the according-to-protocol group were protected from persistent HPV 16 infection, whereas in the placebo group 41 individuals acquired HPV 16 DNA, nine of whom also had HPV-related CIN. ${ }^{2}$ Equally encouraging data were reported for the GSK vaccine (a baculovirus-derived HPV 16/18 L1 VLP) at the 21st International Papillomavirus Workshop in Mexico City in March 2004. In a double-blind, placebo-controlled efficacy study, in the according-to-protocol group 100\% of vaccinees were protected against persistent HPV 16 or 18 infection. Importantly, in the intention-to-treat group in this study, $94 \%$ of vaccinees were protected despite an incomplete vaccination regime. The results from the various trials strongly indicate that vaccination of previously uninfected women using HPV 16 or 18 L1 VLPs is safe and protective, preventing HPV 16 or 18 infection (as measured by acquisition of HPV 16 or 18 DNA) and the development of low-grade intra-epithelial lesions.

However, there are some important issues that must be considered. A key question concerns the duration of the protection induced by these vaccines. Will we need frequent booster immunisations? Preliminary data from several Phase II trials indicate that antibody levels fall from the peak levels achieved after immunisation to a low but measurable level that persists for at least 36 months postvaccination. This is encouraging because it mirrors the situation in animal models where protection is long lasting, despite low levels of circulating antibody. However, the data from the trials cover a relatively short time span and, in reality, we do not know how long the protection induced by L1 VLPs will last. Will exposure to virus postvaccination act as a natural booster? There is no unequivocal evidence for or against, but the preliminary indications from the trials are that this is probably not the case. It is assumed (and the natural infections in animals support this) that these vaccines will only be effective preexposure to virus. Genital HPV infection is usually sexually transmitted and immunisation must therefore precede the sexual debut, implying that the target population for vaccination will be 9-10-year-old prepubertal girls. This may be difficult for cultural and social reasons, particularly in developing countries.

The protection provided by the L1 VLP vaccines appears to be type specific. Thus immunisation with HPV $16 \mathrm{~L} 1$ VLPs protects against HPV 16 infection but not against any of the 34 other genital HPVs. Similarly, HPV 18 L1 VLPs protect against HPV 18 infection but no other HPV. The current generation of VLP vaccines contain only HPV 16 and 18 and, assuming that HPV 16 accounts for 50-60\% and HPV $18 \quad 10-12 \%$ of cervical cancer cases, even in the best scenario (with $100 \%$ vaccine coverage of the target population) only $60-70 \%$ of cervical cancers would be prevented. The cumulative prevalence of HPV types in cervical cancer is illustrated in Figure 1, and it is clear that increasing the number of types in the vaccine (e.g. $16,18,31,45,59)$ would prevent more than $80 \%$ of cancers, but to prevent more than $90 \%$ of cancers at least a further six types would need to be added. ${ }^{3}$ 


\section{Editorial}

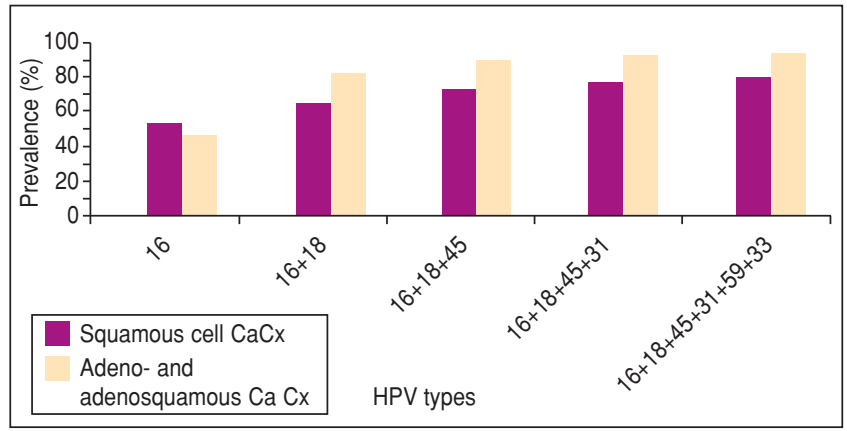

Figure 1 Cumulative prevalence of human papillomavirus (HPV) types in women with cervical cancer. $\mathrm{CaCx}$, cancer of the cervix

Three questions are often posed about polyvalent HPV vaccines, namely:

1. If HPV types 31 and 45 and/or others are included as well as 16 and 18, will we get similar titres of antibody to each HPV type and achieve $80-90 \%$ prevention of cancer? The answer to this question is 'probably yes', since in a Phase I trial examining the immunogenicity and safety of an HPV 16/18/6/11 quadrivalent vaccine the vaccine recipients developed serum antibody to each VLP component and at comparable titres. ${ }^{4}$

2. Will we need different 'cocktails' of HPV types for different populations? There is no answer to this question at present. The HPV type distribution in cervical cancers is generally consistent worldwide but several reports using highly sensitive HPV detection and typing systems have found geographical variability. ${ }^{3}$

3. If we control types that are currently the most common, will other rarer types take their place? This is another unanswerable question at the present time but some indication may come from the Phase III trials of the 16/18 VLP vaccines.

What is clear is that if the current HPV prophylactic vaccines are introduced for mass immunisation in countries with effective cervical cancer screening programmes, such as the UK, these programmes will have to continue unless significant cross-protection is induced by VLP immunisation (and this seems an unlikely scenario). However if vaccines including five or six types (e.g. $16 / 18 / 31 / 45 / 59$ ) were to be licensed and vaccine coverage in the target population was high enough, this might prove sufficiently effective for screening to no longer be considered cost-effective.

\section{Therapeutic vaccines}

Whilst the development of prophylactic vaccination against HPV is exciting, realistically these interventions are at least a decade away. However, even if several decades must elapse before any effects will be evident, the need to develop effective immunotherapies remains a priority. The induction of strong, cell-mediated (as opposed to antibody) responses is certainly central to any therapeutic vaccine strategy and may be critical for long-term immunity in prophylaxis. It is important to define 'therapeutic' in this context and there are three possible scenarios:

1. A vaccine designed to be effective post-exposure to HPV.

2. A vaccine that could be effective against low-grade CIN.

3. A vaccine for high-grade $\mathrm{CIN}$ and cancer.

The antigenic targets in the first two scenarios might be identical but only the oncoproteins E6 and E7 are possible targets for the third scenario, since these are the only viral proteins that will be expressed in all high-grade lesions or cancer (Figure 2).

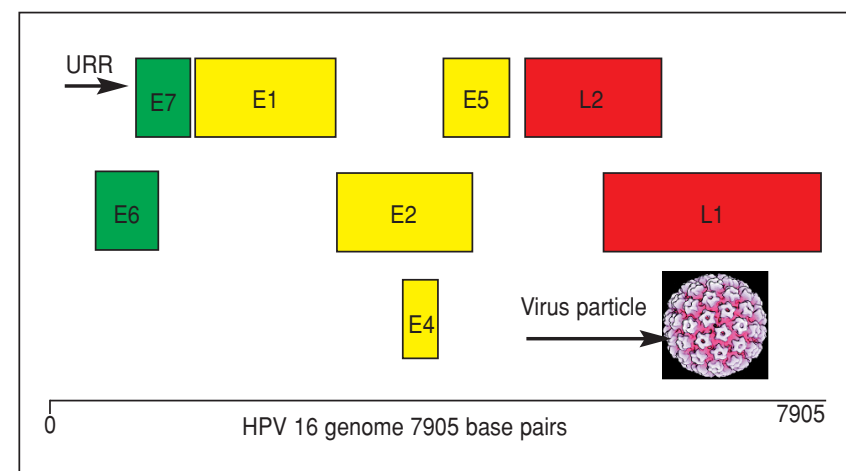

Figure 2 Diagrammatic representation of the gene organisation of the human papillomavirus (HPV) 16 virus. E genes are early genes important for virus growth. E6 and E7 are oncogenes and often are the only genes expressed in high-grade cervical intra-epithelial neoplasia and cancer. Therapeutic vacines for cancers must be targeted to the products of these Then $L$ gene products make up the virus coat or capsid. L1 is the genes. Lene products make up the virus coat or capsid. Ll is the major coat protein. A high-resolution computer image of the virus particle is arrowed. URR, upstream regulatory region

Post-exposure vaccines are worth more than a passing consideration. The communities with the highest incidence of cervical cancer are predominantly in the developing world, and in many societies immunising young girls before the sexual debut may not be easy for social and/or cultural reasons but immunising women would pose fewer problems. HPV testing, if adopted as the primary screening modality, could identify infection as opposed to clinical disease in many individuals; a postexposure vaccine would have a place in the management of such women. In the dog and rabbit, immunisation with vaccines encoding E1 or E2 genes modified to increase antigen expression protects against challenge with live virus. Animals challenged with virus and subsequently immunised with an E2 vaccine either do not develop warts or established lesions regress, indicating that this may be both a post-exposure vaccine and immunotherapy for lowgrade disease. These data from animal models have significant implications for the design of HPV vaccines and suggest that inclusion of an early protein such as E2 together with L1 would provide both prophylaxis and protection post-exposure and could be a second-generation vaccine.

High-grade and low-grade CIN should be considered separately when discussing therapeutic vaccines. Lowgrade CIN is homogeneous and the lesions are genetically stable; in an immunocompetent individual an effective therapeutic vaccine such as an E2 vaccine should result in lesion clearance and no recurrence. CIN $2 / 3$ is heterogeneous, and the lesions are genetically unstable with the probability that immune parameters of importance will be disregulated. In view of this scenario it is distinctly possible that there will be a spectrum of responses to E6/E7 vaccination in patients with high-grade disease ranging from complete through partial to no clearance of the clinical disease - indeed this is what has been observed in the Phase I/II trials that have been carried out to date. 5 Therapeutic vaccines for high-grade and malignant anogenital disease have been disappointing to date, and some significant scientific developments are needed if these are to have clinical utility.

\section{Five-year view}

HPV VLP vaccines have made cervical cancer, in theory, a preventable disease and the next 5 years should see the licensing of the first generation of these vaccines. All the evidence suggests that, for efficacy, the vaccines will have to be delivered prior to the sexual debut and, in 
reality, this means that prepubertal girls will form the vaccinated population. The take up of such vaccines will depend upon social attitudes, public health policies and economics. Such a scenario prompts a number of currently unanswerable questions, namely: How well will a vaccine against a sexually transmitted agent which protects against a disease that may develop in 30 years' time will be accepted? How extensive will the coverage be if 9-10-year-old girls are the vaccinated group? And, finally, since vaccination might not mean the elimination of cervical cancer screening programmes, will governments and/or insurance providers pay for vaccination and screening?

Statements on funding and competing interests

Funding. The author has received funding via a GlaxoSmithKline Research Collaboration.

Competing interests. The author is a consultant for GlaxoSmithKline Immunotherapeutics.
Margaret A Stanley, MB, PhD

Professor of Epithelial Biology, University of Cambridge. Department of Pathology, Tennis Court Road, Cambridge CB2 1QP, UK. Tel: +44 (0) 1223 333736. Fax: +44 (0) 1223 333730.E-mail:mas1001@cam.ac.uk

References

1 Schiffman M, Kjaer SK. Natural history of anogenital papillomavirus infection and neoplasia. J Natl Cancer Inst Monogr 2003; 31: 14-19.

2 Koutsky LA, Ault KA, Wheeler CM, et al. A controlled trial of a human papillomavirus type 16 vaccine. $N$ Engl J Med 2002; 347: 1645-1651.

3 Bosch FX, Sanjose S. Human papillomavirus and cervical cancerburden and assessment of causality. J Natl Cancer Inst Monogr 2003; 31: $3-11$

4 Brown DR, Ault KA, Wheeler CM, et al. A dose ranging study of the safety and immunogenicity profiles of a quadrivalent HPV (types 6 , 11,16 and 18) L1 VLP candidate vaccine in young healthy women. Abstract (0-51) presented at the 19th International Papillomavirus Workshop, Florianopolis, Brazil, 1-7 September 2001.

5 Stanley MA. Genital human papillomavirus infections - current and prospective therapies. J Natl Cancer Inst Monogr 2003; 31: 117-124.

\section{LETTERS TO THE EDITOR}

Letters to the Editor are welcome and generally should not exceed 600 words or cite more than five references. For comments on material published in the most recent issue of the Journal, correspondence should be received within 4 weeks of dispatch of that Journal to be in time for inclusion in the next issue. When submitting letters correspondents should include their job title, a maximum of two qualifications and their address(es). A statement on competing interests should also be submitted for all letters. Letters may be submitted to the Editor or the Journal Editorial Office (details on page 205).

\section{But is she positive?}

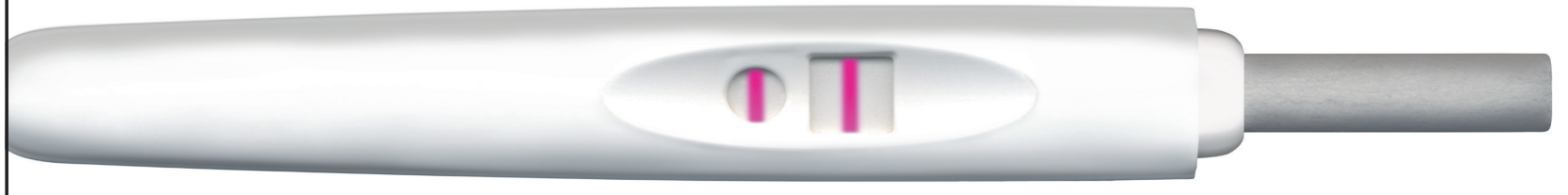

Your next patient is pregnant but doesn't want to be.

Where next? As a registered charity since 1968, bpas has offered affordable abortion care for women. We provide almost 50,000 abortions a year (including service agreements) and can offer all the professional help your patient needs.

bpas has a nationwide network of clinics and consultation centres. There are no long waits for appointments. We can offer a choice of times, clinics and procedures. All it takes to arrange an appointment is one call to the bpas Actionline on 08457304030.

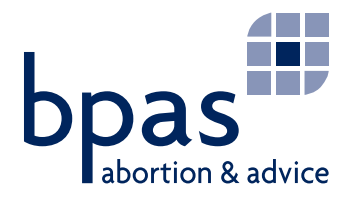

ACTIONLINE 08457304030

bpas positively the best service 